\begin{tabular}{|c|l|}
\hline Title & Three Dimensional Microscopic Elemental A naly sis Using an A utomated High-Precision Serial Sectioning System \\
\hline Author(s) & $\begin{array}{l}\text { Fujisaki, Kazuhiro; Y okota, Hideo; Furushiro, Naomichi; Komatani, Shintaro; Ohzawa, Sumito; Sato, Y oshimichi; } \\
\text { Matsunaga, Dai suke; Himeno, Ryutaro; Higuchi, Toshiro; Makinouchi, A kitake }\end{array}$ \\
\hline Citation & $\begin{array}{l}\text { Microscopy and Microanalysis, 17(2), 246-251 } \\
\text { https:/doi.org/L0.1017/S143192761009450X }\end{array}$ \\
\hline Issue Date & 2011-0401 \\
\hline Doc URL & http://hdl.handle.net/2115/48720 \\
\hline Rights & Copyright $\odot$ Microscopy Society of A merica 2011 \\
\hline Type & article \\
\hline File Information & MaM17-2_246-251.pdf \\
\hline
\end{tabular}

Instructions for use 


\title{
Three-Dimensional Microscopic Elemental Analysis Using an Automated High-Precision Serial Sectioning System
}

\author{
Kazuhiro Fujisaki, ${ }^{1,2}$ Hideo Yokota, ${ }^{1,2,3, \star}$ Naomichi Furushiro, ${ }^{2,4}$ Shintaro Komatani, ${ }^{5}$ \\ Sumito Ohzawa, ${ }^{5}$ Yoshimichi Sato, ${ }^{5}$ Daisuke Matsunaga, ${ }^{5}$ Ryutaro Himeno, ${ }^{3}$ Toshiro Higuchi, ${ }^{3,6}$ \\ and Akitake Makinouchi ${ }^{1,7}$ \\ ${ }^{1}$ Division of Human Mechanical Systems and Design, Graduate School of Engineering, Hokkaido University, \\ N13 W8, Kita-ku, Sapporo 060-8628, Japan \\ ${ }^{2}$ Bio-Research Infrastructure Construction Team, RIKEN, 2-1, Hirosawa, Wako, Saitama 351-0198, Japan \\ ${ }^{3}$ Biomechanical Simulation Research Team, RIKEN, Wako, Saitama 351-0198, Japan \\ ${ }^{4}$ Department of Mechanical Engineering, Faculty of Engineering Science, Kansai University, 3-3-35, \\ Yamate-cho, Suita, Osaka 564-8680, Japan \\ ${ }^{5}$ Horiba, Ltd., 2, Miyanohigashi, Kisshoin, Minami-ku, Kyoto 601-8510, Japan \\ ${ }^{6}$ Department of Precision Engineering, The University of Tokyo, 7-3-1 Hongo, Bunkyo-ku, Tokyo 113-8656, Japan \\ ${ }^{7}$ VCAD System Research Program, RIKEN, 2-1, Hirosawa, Wako, Saitama 351-0198, Japan
}

\begin{abstract}
The elemental composition and microscopic-level shape of inclusions inside industrial materials are considered important factors in fracture analytical studies. In this work, a three-dimensional (3D) microscopic elemental analysis system based on a serial sectioning technique was developed to observe the internal structure of such materials. This 3D elemental mapping system included an X-ray fluorescence analyzer and a highprecision milling machine. Control signals for the X-ray observation process were automatically sent from a data I/O system synchronized with the precision positioning on the milling machine. Composite specimens were used to confirm the resolution and the accuracy of 3D models generated from this system. Each of the two specimens was composed of three metal wires of $0.5 \mathrm{~mm}$ diameter braided into a single twisted wire that was placed inside a metal pipe; the pipe was then filled with either epoxy resin or Sn. The milling machine was used to create a mirror-finish cross-sectional surface on these specimens, and elemental analyses were performed. The twisted wire structure was clearly observed in the resulting 3D models. This system enables automated investigation of the $3 \mathrm{D}$ internal structure of materials as well as the identification of their elemental components.
\end{abstract}

Key words: serial sectioning, elemental mapping, microscopic structure, precision cutting, three-dimensional model, internal structure, automation

\section{INTRODUCTION}

The distribution of inclusions and their amounts in industrial materials are important to evaluate the quality of materials in metal manufacturing processes. An origin of crack propagation resulting in a material fracture commonly occurs at the boundary regions between inclusions and the base metal. The distribution and location of inclusions as well as their three-dimensional (3D) shapes are useful information in fracture analysis. X-ray computed tomography (CT) systems are widely used to observe the 3D internal structure of materials constructed with complex components. Recently, high-energy X-rays such as synchrotron X-rays have been used for high-resolution observations of the internal structure of metal materials. In particular, synchrotron X-rays have been applied to measure the internal structure of aluminum alloys at the microscopic scale (Baruchel et al., 2006), as well as fatigue crack propagation (Khor et al., 2006; Zhang et al., 2009).

Lauridsen et al. (2006) investigated the process of recrystallization in cold-rolled copper by means of $3 \mathrm{D}$

Received June 15, 2010; accepted November 11, 2010

${ }^{\star}$ Corresponding author. E-mail: hyokota@riken.jp
X-ray diffraction microscopy. However, for cases in which a single material is composed of several heavy metals with high X-ray absorption, X-ray CT methods cannot easily provide internal structure information. Instead, such materials have been analyzed by means of serial sectioning techniques, in which the cross sections of the materials' surfaces are observed by microscopic imaging; the resulting images are then aligned to form a 3D internal structure model (Alkemper \& Voorhees, 2001).

Various analytical processes can be easily applied to the sectional surfaces in serial sectioning methods. The serial sectioning method requires the surface of the sample material to be mirror-like, flat, and composed of optically fine planes for metallographic observations. Generally, polishing processes are used to create a mirror-like specimen surface, but such processes require an immense amount of time and effort. Such polishing has been used to create the mirror-like surfaces in the serial sectioning observation of high X-ray absorption materials including Sn solders (Sidhu \& Chawla, 2004; Dudek \& Chawla, 2008). Additionally, Alkemper and Voorhees (2001) used a milling machine to prepare soft metal surfaces for serial section imaging and presented an image alignment method for 3D constructions. Since these 3D re- 


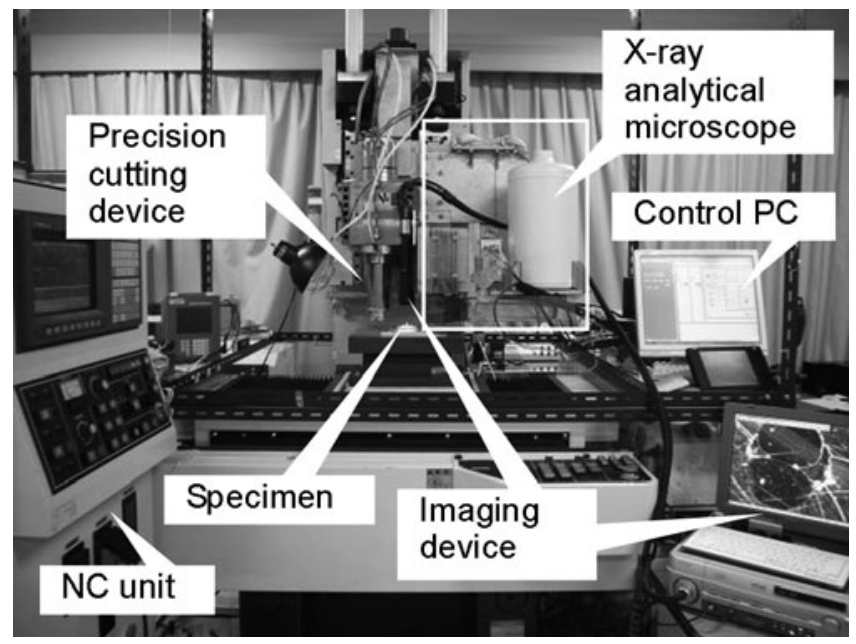

Figure 1. Photograph of Riken micro slicer system RMSS-003 modified with an X-ray analytical microscope.

construction studies require high-resolution surface imaging and the rapid acquisition of many serial section images, a high-precision and rapid cutting technique should be developed for serial sectioning methods.

For years, the authors have been developing 3D internal structure microscopes to observe biological samples on the microscopic scale [Riken Micro Slicer System: RMSS-001, 002 (Yokota et al., 2001, 2002)]. A specimen is positioned in the microscope, and then a very thin layer of the specimen is removed by a slicing process. After slicing, the newly created surface can be observed by two-dimensional (2D) optical imaging and also can be subjected to various analyses. When a series of automated slicing and 2D imaging steps are employed, the resulting 2D images can be aligned to construct a 3D internal structure of the material. In previous studies, the authors developed a 3D internal structure observation system that incorporates a precision milling machine instead of the slicing tool used to machine hard materials such as metals (Furushiro et al., 2008). The system automatically generated 3D structures for the shapes of inclusions in bearing steels with high-resolution imaging at the submicron scale (Fujisaki et al., 2010). However, these observations could not classify the kinds of inclusions that were present in the materials. In this study, an elemental analysis process was introduced into the serial sectioning method to obtain the distribution patterns of components and subsequently identify their elements. The accuracy of 3D internal structure observations with the elemental mapping was evaluated from the reconstruction scale error in the observation of composite metal specimens.

\section{Materials and Methods}

\section{D Observation System}

The 3D internal structure microscope (Riken Micro Slicer System-003: RMSS-003) was developed on the basis of a high-precision milling machine UVM-350(J) (Toshiba Machine Co., Ltd., Shizuoka-ken, Japan) as shown in Figure 1

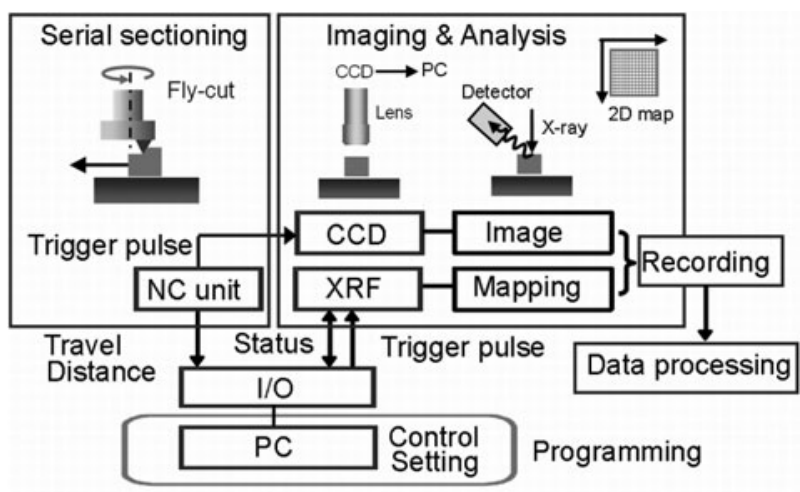

(a) Internal structure observation process

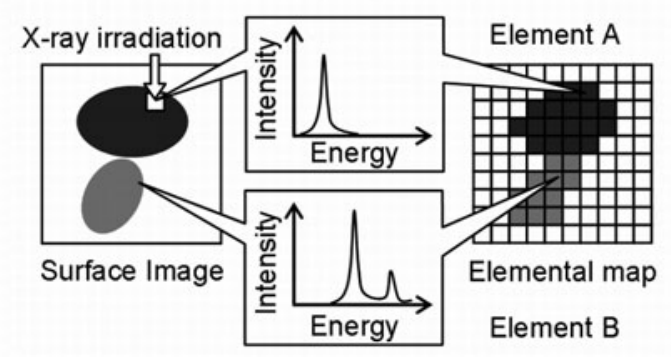

(b) Elemental mapping

Figure 2. Block diagrams illustrating the processes of (a) internal structure acquisition in serial sectioning and (b) elemental mapping on the RMSS-003.

(Furushiro et al., 2008). The $z$-axis of the machine has a large surface plate to attach a milling spindle, which can turn rapidly at up to 30,000 revolutions per minute by means of an air bearing. The machine also features observation devices such as optical microscopes. Each $x, y$, and $z$ table can be positioned precisely at a high-resolution feedback scale of $0.1 \mu \mathrm{m}$. Precision positioning was achieved with a programmable numerical control (NC) system in the milling machine. Figure 2a shows an overview of the system with control devices. Information about the $x, y$, and $z$ table moving distances, acquired as a pulsed-wave pattern readout from the NC unit, is collected by the data I/O system PXI (National Instruments Co., Ltd., Austin, TX, USA) and is processed with LabVIEW software. Elements present in the specimens were detected by X-ray fluorescence analysis. Excitation fluorescence $\mathrm{X}$-rays generated from incident $\mathrm{X}$-rays irradiating the surface of the specimen were detected under atmospheric pressure conditions. A 3D microscopic elemental analysis system was constructed from the RMSS-003 equipped with an X-ray generator and a fluorescence detector, which were separated from an X-ray fluorescence analyzer (XGT-5000: X-ray analytical microscope, Horiba, Ltd., Kyoto, Japan). X-rays were generated from an Rh target and a parallel beam with $10 \mu \mathrm{m}$ diameter was formed by a monocapillary in the X-ray guide tube (Ohzawa et al., 2004). The X-ray irradiation periods and detecting periods (i.e., the exposure time) were controlled by the LabVIEW software using external signals from the I/O system, and $\mathrm{X}$-ray scanning measurements were performed on the ma- 


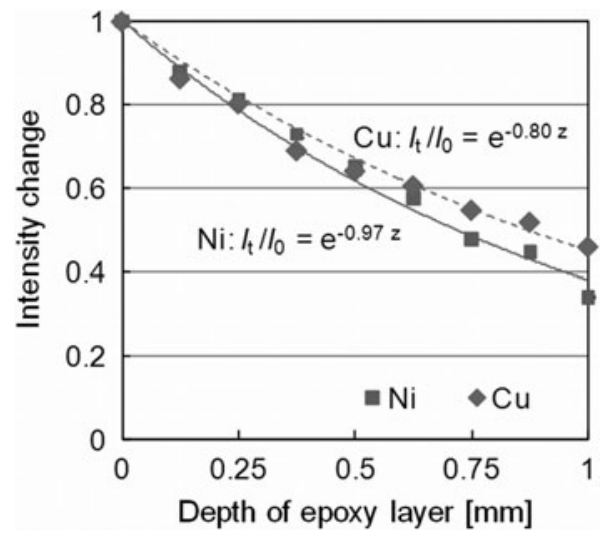

Figure 3. X-ray absorption of epoxy resin in X-ray fluorescence measurements of epoxy-filled stepped ditches created in $\mathrm{Cu}$ and Ni samples.

chine tables. The intensity versus X-ray energy profile (i.e., the spectrum) of the fluorescence X-rays was detected at all points of the positions in Figure $2 \mathrm{~b}$. The resulting spectra were characteristic of elements present in the specimens and were thus used to identify the elements. The mapping pattern of each element was obtained as a 2 D image $(256 \times$ 256 or $512 \times 512$ pixels) on a cross-sectional surface of specimen.

The resolution of 3D reconstructions obtained by serial sectioning depends on the magnifying power of the microscope on each section (in the $x$ - and $y$-planes) and on the cutting depth (in the $z$-plane). In the X-ray measurements, the X-ray penetration depth affected the resolution in the $z$-plane. The ratio of intensities between the initial value $I_{\text {in }}$ of the incident $\mathrm{X}$-rays and the value penetrated through depth $z\left(I_{z}\right)$ is represented by equation (1):

$$
I_{z} / I_{\text {in }}=e^{-\mu z}
$$

In this equation, $\mu$ is the X-ray absorption coefficient, of which the value is determined from the wavelength of transmitted X-rays and the type of absorption material. In this analysis, the wavelengths of the incident $\mathrm{X}$-rays and the excitation fluorescence X-rays generated from the measured materials differed, and thus the values of $\mu$ also differed. The ratio of intensities between the X-rays with the absorption material $\left(I_{t}\right)$ and those in the absence of material $\left(I_{0}\right)$ for materials with absorption coefficients $\mu_{i}$ and penetration distances $l_{i}$ is denoted by equation (2):

$$
I_{t} / I_{0}=e^{-\sum \mu_{i} l_{i}}
$$

In this study, these absorption characteristics were investigated in X-ray penetration tests. The specimens were made of $\mathrm{Cu}$ and $\mathrm{Ni}$ and had stepped ditches from 0 to $2 \mathrm{~mm}$ depth by $0.125 \mathrm{~mm}$ step. Each stepped ditch was filled with epoxy resin as an absorption material. Incident $\mathrm{X}$-rays were transmitted through the epoxy region, and then the excited X-rays from the metal materials were transmitted through the epoxy region again to be detected on the reflection side of the specimen surface. Figure 3 shows the change in X-ray intensity as a function of epoxy resin thickness. These values were measured under incident $\mathrm{X}$-rays generated at $50 \mathrm{kV}$ and $1 \mathrm{~mA}$ in the X-ray fluorescence analyzer. The results for both $\mathrm{Cu}$ and $\mathrm{Ni}$ specimens showed that the X-ray intensity decreased logarithmically with increasing absorption depth, though the rate of decrease was larger for $\mathrm{Ni}$ than for $\mathrm{Cu}$. This difference indicated that the $\mathrm{X}$-ray absorption coefficients depended on the wavelength of fluorescence $\mathrm{X}$-rays generated from the $\mathrm{Cu}$ and $\mathrm{Ni}$, as expressed in equation (2). As epoxy thickness increased to $0.5 \mathrm{~mm}$, the detected X-ray intensity decreased to about $50 \%$ of the value of the X-ray intensity detected without the epoxy layer. These results show that the X-rays were able to penetrate these radiolucent materials to depths of several hundreds of microns. In contrast, when Sn was used in place of the epoxy resin for shielding, the intensity of the fluorescence X-rays could not be detected at Sn thicknesses as small as $0.125 \mathrm{~mm}$.

The 3D distributions of each element divided in voxel regions were independently and quantitatively obtained from $2 \mathrm{D}$ elemental mapping. For compound regions in the material, in which more than one element was present per voxel, or for cases in which particles smaller than the voxel volume were present, the mass fraction of each element in the voxel was quantitatively estimated from a reference voxel containing $100 \%$ of the element. In this study, composite specimens were fabricated from pure metals. Therefore, each pixel in the $2 \mathrm{D}$ elemental mapping data was approximated to contain $100 \%$ of the predominant element observed in that pixel. The pixels containing elemental information were classified into 8 -bit (0 to 255 ) grayscale images. The 3D volume model used for elemental mapping was constructed by the rendering software V-Cat (Riken, VCAD system research program, distributed as freeware) using the 2D labeling images. For 3D finite element analysis, the VCAD system software created 3D surface models as stereolithography format files and as original 3D volume format files.

\section{Experimental Procedures}

Composite specimens were used to evaluate the analyzing process controlled by the precision positioning in the system. Two types of specimens containing embedded substances made of materials with low or high degrees of X-ray absorption were prepared by means of the following process. First, metal wires made of $\mathrm{Ag}, \mathrm{Zn}$, and $\mathrm{Sn}$ of $0.5 \mathrm{~mm}$ diameter were braided into a single twisted wire and were placed inside an $\mathrm{Al}$ pipe of $5 \mathrm{~mm}$ external diameter and $3 \mathrm{~mm}$ internal diameter. The remaining volume inside the pipe was filled with epoxy resin (specimen A). Additionally, metal wires made of $\mathrm{Ti}, \mathrm{Cu}$, and $\mathrm{Ni}$ of $0.5 \mathrm{~mm}$ diameter were braided into a single twisted wire and were placed inside a Ti pipe of $4 \mathrm{~mm}$ external diameter and $3 \mathrm{~mm}$ internal diameter. The remaining volume inside this pipe was filled with melted Sn (specimen B). Each of these two specimens was fixed on a duralumin plate and placed on the $x-y$ table of the RMSS-003.

Measured points were located on a cross-sectional surface of each specimen, which was processed to a mirror 

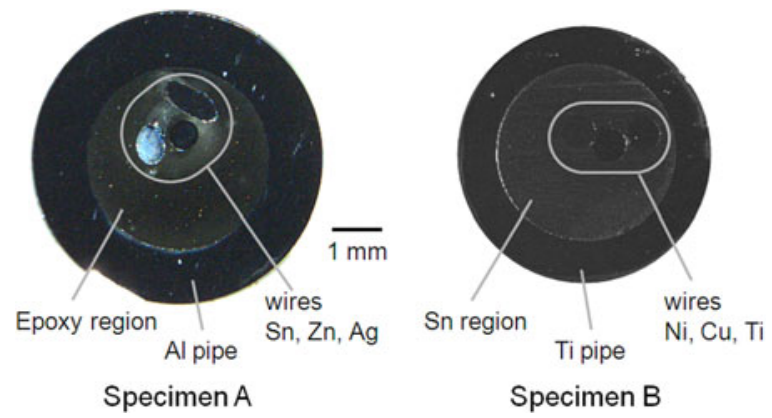

Figure 4. Cross-sectional surface image of specimens A (Ag, Zn, and $\mathrm{Sn}$ braided wire inside an $\mathrm{Al}$ pipe filled with epoxy resin) and $\mathrm{B}(\mathrm{Ti}, \mathrm{Cu}$, and $\mathrm{Ni}$ braided wire inside a Ti pipe filled with $\mathrm{Sn})$.

finish by precision cutting as shown in Figure 4. The surface images had little contrast in the stereomicroscope imaging because all components were finished as a mirror-like flat surface by high-precision cutting. The boundary of components, particularly in the metals in specimen $B$, was difficult to recognize in the surface image. Table 1 lists the conditions used for precision cutting and subsequent X-ray measurements. Cutting tools made from single-crystal diamond are generally used for precision cutting in such applications as mold manufacturing processes. The high-speed milling was conducted with the diamond tool at a cutting speed of $942 \mathrm{~m} / \mathrm{min}, 5 \mu \mathrm{m}$ feed under dry conditions with air blowing. The cutting depth for each section was $10 \mu \mathrm{m}$, and each X-ray measurement to detect the $2 \mathrm{D}$ map was performed by 20 slices (the distance of each mapping image was $200 \mu \mathrm{m}$ in depth). The X-ray measurement region was set at $5.1 \times 5.1 \mathrm{~mm}$. The area was divided in $256 \times 256$ points for creating the $2 \mathrm{D}$ mapping images. The image resolution was $20 \times 20 \mu \mathrm{m}$ for a pixel, which was larger than X-ray irradiation spot size of $10 \mu \mathrm{m}$ diameter. Element information of the pixel was represented by measured area of the X-ray irradiation spot.

\section{Results}

Figure 5 shows the 2D elemental mapping images of a $5.1 \times$ $5.1 \mathrm{~mm}$ region of the cross section of specimen A. The offset in positioning in the $x y$ plane between these sectional images was lower than the imaging resolution $(20 \mu \mathrm{m})$, which was confirmed from $\mathrm{Al}$ distributions presented in the

Table 1. Measurement Conditions for Serial Sectioning and Elemental Mapping of Specimens A and B.

$\begin{array}{ll}\text { Cutting tool } & \text { Single-crystal diamond } \\ \text { Cutting speed } & 942 \mathrm{~m} / \mathrm{min} \\ \text { Feed } & 5 \mu \mathrm{m} / \mathrm{rev} \\ \text { Cutting depth } & 10 \mu \mathrm{m} \\ \text { Tube voltage, current } & 50 \mathrm{kV}, 1 \mathrm{~mA} \\ \text { Exposure time } & 80 \mathrm{~ms} / \text { point } \\ \text { Mapping image size } & 256 \times 256 \text { pixels } \\ \text { Resolution }(x, y, z) & 20,20,200 \mu \mathrm{m} / \text { pixel }\end{array}$

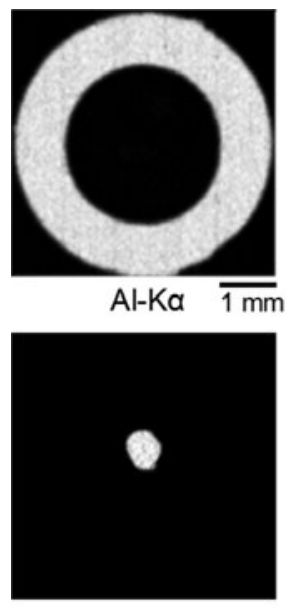

$\mathrm{Zn}-\mathrm{Ka}$

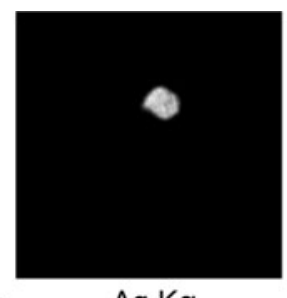

$\mathrm{Ag}-\mathrm{Ka}$

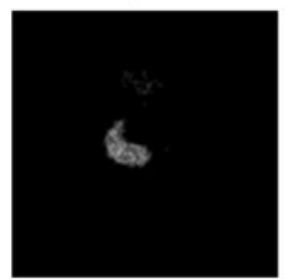

$\mathrm{Sn}-\mathrm{Ka}$

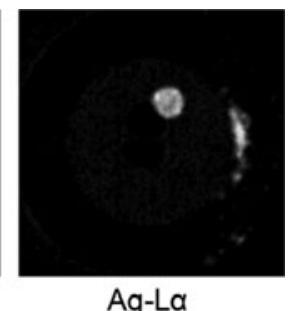

$\mathrm{Ag}-\mathrm{L} \alpha$

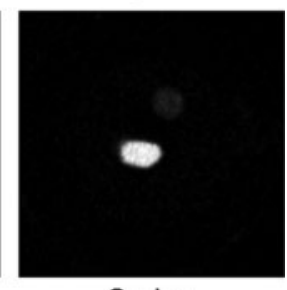

$\mathrm{Sn}-\mathrm{Lq}$
Figure 5. 2D elemental mapping image of each component on a cross-sectional surface of specimen A.

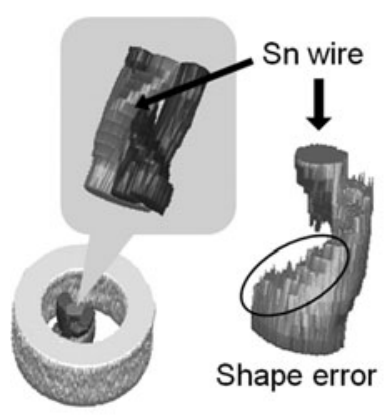

(a) Specimen $\mathrm{A}$

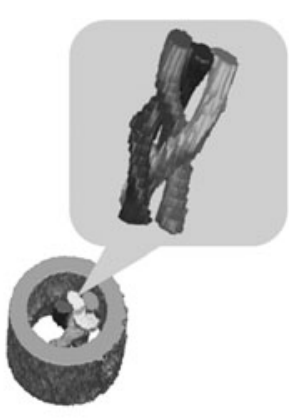

(b) Specimen B
Figure 6. 3D models of (a) the whole material and Sn wire structure in specimen A and (b) the twisted wire structure in specimen B.

sectional surfaces. The circular regions of $\mathrm{Al}$ showed no offset between the images. 3D surface models of each component were constructed from the $2 \mathrm{D}$ elemental mapping images of Al-K $\alpha, \mathrm{Ag}-\mathrm{K} \alpha, \mathrm{Zn}-\mathrm{K} \alpha$, Sn- $\mathrm{L} \alpha$ of 12 cross sections of specimen A. The boundaries of each elemental component could be recognized from the background-subtracted $\mathrm{X}$-ray intensity. Figure 6a shows the 3D models of the twisted wire of specimen A. Each wire was clearly observable even within the space confines of the Al pipe. However, the boundary of the $\mathrm{Sn}$ wire in the model was expanded relative to the actual size of the wire. Figure $6 \mathrm{~b}$ shows the $3 \mathrm{D}$ model of the wires in specimen $\mathrm{B}$, constructed from 30 cross-sectional images of the specimen acquired in the same manner as that used for specimen A. However, in contrast with the 3D model of specimen $\mathrm{A}$, the 3D model of specimen $B$ did not feature errors in wire sizes. This lack of error can be explained as follows: since the X-rays were unable to deeply penetrate the volume of the Ti pipe filled with Sn, the effects of X-ray transmission were minimized in the $3 \mathrm{D}$ model.

Although a time of about $2 \mathrm{~h}$ was required to obtain a single $2 \mathrm{D}$ image for a $5.1 \times 5.1 \mathrm{~mm}$ region with $256 \times 256$ 
pixels, the NC unit and LabVIEW software automated all the processes associated with the image acquisition, including preparation of the mirror surface and elemental mapping by means of X-ray measurements. This automated system enabled the investigation of the $3 \mathrm{D}$ internal structure of the materials as well as the identification of their component elements.

\section{Discussion}

Although serial sectioning is a destructive observation technique, it can provide valuable information for evaluation of a material's defect distributions based on structural analysis at many levels, from boundaries of material components to the crystallographic structure of the material. A 3D elemental model was constructed from uniform element voxels with 20,20 , and $200 \mu \mathrm{m}$ in serial sectioning and analysis. The voxel resolution in the $z$-direction depends on the cutting depth; to maintain fine-precision cutting in this present apparatus, the minimum cutting depth is about 1 or $2 \mu \mathrm{m}$. However, the elemental analysis included depth information by X-ray penetration, as shown in specimen A with epoxy regions. The precision in the $x$ - and $y$-directions was determined in the scanning process, which can be modified freely to change the feed of the $x-y$ table in the NC unit and the irradiation time controlled by the LabVIEW software. Although the scan resolution can be set below $10 \mu \mathrm{m}$ by the software, the device's X-ray spot size of $10 \mu \mathrm{m}$ limited the resolution achievable in 2D elemental mapping in the present experiments. Microscopic observations with nanometerscale resolution have been reported for energy-dispersive $\mathrm{X}$-ray spectroscopy in scanning electron microscope (SEM) or transmission electron microscope observations of a material cross section prepared by focused ion beam slicing (Schaffer et al., 2007). The high-resolution measurements might be conducted in an SEM, which has higher spatial resolution compared to X-ray fluorescence. The sectioning system is useful for multiscale analysis on several tens of micrometers resolution in several centimeter regions. This method can operate without special environmental conditions such as vacuum or strict temperature controls.

The 3D internal structure microscope system described here can be used to investigate many kinds of materials for which mirror surfaces can be prepared by means of precision cutting processes with diamond tools. However, diamond tools are not appropriate for the precision cutting of some metals (Paul et al., 1996). In particular, mirror surfaces on steel specimens are more effectively created with cBN tools (Fujisaki et al., 2009), with elliptical vibration cutting devices (Moriwaki \& Shamoto, 1995; Shamoto \& Moriwaki, 1999), or by using polishing processes instead of milling processes (Spowart, 2006).

The 2D elemental images contained depth information beneath the surface of the specimens because the X-rays were transmitted through the material's surface. Notably, in Figure $6 \mathrm{a}$, the $3 \mathrm{D}$ model of the $\mathrm{Sn}$ wire was expanded relative to the actual size of the Sn wire, whereas specimen B showed almost no error in wire size in its 3D image. This difference in images arose because the X-rays penetrated the epoxy filling of specimen A more easily than they penetrated the Sn filling of specimen B. The X-rays generated from deep areas were detected without absorption by epoxy shielding. The effect is difficult to eliminate completely. It might be modified by using the all sectional 2D mapping data using some estimation methods with inverse problem, for example, using the difference of continuous mapping images.

Under the present measurement conditions, the X-ray exposure time was too short to allow quantitative investigation of the mass fraction of elements. The fraction shows only the scale of 0 to 5 range to consider $\mathrm{S} / \mathrm{N}$ ratio under the measurement condition. The $2 \mathrm{D}$ elemental mapping data were approximated to contain $100 \%$ of the predominant element observed in that pixel by using an appropriate threshold value to eliminate the noise and the effect of $\mathrm{X}$-rays generated from subsurface depths. The shape error by X-rays transmitted through the epoxy in the case of specimen A was over $200 \mu \mathrm{m}$ deep even when the threshold was set at 5 , which was more than $80 \%$ of the maximum intensity, as shown in Figure 3.

For quality management of industrial materials, decreasing the number of inclusions and driving the locations of such inclusions into the noncritical areas of materials are required. The 3D shape of inclusions, their distribution, and their elemental composition are important information that can be used to estimate the strength of the material with regard to fracture or fatigue processes. The serial sectioning system proposed here can be used for the evaluation of such material properties.

\section{Conclusions}

A 3D microscopic elemental analysis system based on a serial sectioning technique was developed to observe the internal structure of materials. This $3 \mathrm{D}$ elemental mapping system included an X-ray fluorescence analyzer and a highprecision milling machine. Composite specimens including twisted metal wires were used to confirm the resolution and the accuracy of 3D models generated from this system. The twisted wire structure was clearly observed in the resulting 3D models. This system enables automated investigation of the 3D internal structure of materials as well as the identification of their elemental components.

\section{REFERENCES}

Alkemper, J. \& Voorhees, P.W. (2001). Quantitative serial sectioning analysis. J Microsc 201(3), 388-394.

Baruchel, J., Buffiere, J., Cloetens, P., Michiel, M.D., Ferrie, E., Ludwig, W., Maire, E. \& Salvo, L. (2006). Advances in synchrotron radiation microtomography. Scripta Mater 55(1), $41-46$.

Dudek, M.A. \& Chawla, N. (2008). Three-dimensional (3D) microstructure visualization of $\mathrm{LaSn}_{3}$ intermetallics in a novel 
Sn-rich rare-earth-containing solder. Mater Charact 59(9), 1364-1368.

Fujisaki, K., Yokota, H., Furushiro, N., Yamagata, Y., TaniguChi, T., Himeno, R., Makinouchi, A. \& Higuchi, T. (2009). Development of ultra-fine-grain binderless cBN tool for precision cutting of ferrous materials. J Mater Proc Technol 209, 5646-5652.

Fujisaki, K., Yokota, H., Nakatsuchi, H., Yamagata, Y., Nishikawa, T., Udagawa, T. \& Makinouchi, A. (2010). Observation of three-dimensional internal structure of steel materials by means of serial sectioning with ultrasonic elliptical vibration cutting. J Microsc 237 (1), 89-95.

Furushiro, N., Үoкотa, H., Fujisaki, K., Yamagata, Y., Кокubo, M., Himeno, R., Makinouchi, A. \& Higuchi, T. (2008). Three-dimensional internal information acquisition system based on consecutive precision machining and cross-sectional observation-Development of the system and its applications. Proceedings of ASPE 2008 Annual Meeting, pp. 180-183. Raleigh, NC: American Society for Precision Engineering.

Khor, K., Buffiere, J., Ludwig, W. \& Sinclair, I. (2006). High resolution X-ray tomography of micromechanisms of fatigue crack closure. Scripta Mater 55(1), 47-50.

Lauridsen, E.M., Schmidt, S., Nielsen, S.F., Margulies, L., Poulsen, H.F. \& Juul Jensen, D. (2006). Non-destructive characterization of recrystallization kinetics using threedimensional X-ray diffraction microscopy, Scripta Mater 55(1), 51-56.

Moriwaki, T. \& Sнамото, E. (1995). Ultrasonic elliptical vibration cutting. CIRP Ann-Manuf Techn 44(1), 31-34.

Ohzawa, S., Komatani, S. \& OвоRi, K. (2004). High intensity monocapillary X-ray guide tube with 10 micrometer spatial resolution for analytical X-ray microscope. Spectrochim Acta B 59(8), 1295-1299.

Paul, E., Evans, C.J., Mangamelli, A., McGlauflin, M.L. \& Polvani, R.S. (1996). Chemical aspects of tool wear in single point diamond turning. Precis Eng 18, 4-19.

Schaffer, M., Wagner, J., Schaffer, B., Schmied, M. \& MulDERs, H. (2007). Automated three-dimensional X-ray analysis using a dual-beam FIB. Ultramicroscopy 107(8), 587-597.

Shamoto, E. \& Moriwaki, T. (1999). Ultrasonicprecision diamond cutting of hardened steel by applying ultrasonic elliptical vibration cutting. Ann CIRP 48, 441-444.

Sidhu, R.S. \& Chawla, N. (2004). Three-dimensional microstructure characterization of $\mathrm{Ag}_{3} \mathrm{Sn}$ intermetallics in Sn-rich solder by serial sectioning, Mater Charact 52 (3), 225-230.

Spowart, J.E. (2006). Automated serial sectioning for 3-D analysis of microstructures. Scripta Mater 55, 5-10.

Yokota, H., Kawaguchi, R., Nakamura, S., Makinouchi, A., Higuchi, T. \& Yabe, Y. (2001). 3-dimensional digitizing for the biological sample using a 3-dimensional internal structure microscope. Proc. 2001 Bioengineering Conference ASME, Vol. 50, pp. 217-218. New York: American Society of Mechanical Engineers.

Yokota, H., Nakamura, S., Kawaguchi, R., Makinouchi, A., Yabe, H. \& Higuchi, T. (2002). Destructed imaging of biological sample using a 3 dimension internal structure microscope. Med Imaging Technol 20(6), 660-665 (in Japanese).

Zhang, H., Toda, H., Qu, P.C., Sakaguchi, Y., Kobayashi, M., Uesugi, K. \& Suzuki, Y. (2009). Three-dimensional fatigue crack growth behavior in an aluminum alloy investigated with in situ high-resolution synchrotron X-ray microtomography. Acta Mater 57(11), 3287-3300. 